\title{
The Korean Society for Neuro-Oncology (KSNO) Guideline for WHO Grade III Cerebral Gliomas in Adults: Version 2019.01
}

\author{
Young Zoon Kim ${ }^{1}$, Chae-Yong Kim², Jaejoon Lim³ ${ }^{3}$ Kyoung Su Sung ${ }^{4}$, Jihae Lee ${ }^{5}$, Hyuk-Jin Oh ${ }^{6}$, Seok-Gu Kang ${ }^{7}$, Shin-Hyuk Kang ${ }^{8}$, \\ Doo-Sik Kong ${ }^{9}$, Sung Hwan Kim ${ }^{10}$, Se-Hyuk Kim ${ }^{11}$, Se Hoon Kim² ${ }^{12}$, Yu Jung Kim ${ }^{13}$, Eui Hyun Kim², In Ah Kim ${ }^{14}$, Ho Sung Kim ${ }^{15}$, \\ Tae Hoon Roh ${ }^{11}$, Jae-Sung Park ${ }^{16}$, Hyun Jin Park ${ }^{17}$, Sang Woo Song ${ }^{18}$, Seung Ho Yang ${ }^{19}$, Wan-Soo Yoon ${ }^{20}$, Hong In Yoon ${ }^{21}$, \\ Soon-Tae Lee ${ }^{22}$, Sea-Won Lee ${ }^{23}$, Youn Soo Lee ${ }^{24}$, Chan Woo Wee ${ }^{14}$, Jong Hee Chang ${ }^{7}$, Tae-Young Jung ${ }^{25}$, Hye Lim Jung ${ }^{26}$, \\ Jae Ho Cho ${ }^{21}$, Seung Hong Choi ${ }^{27}$, Hyoung Soo Choi ${ }^{28}$, Je Beom Hong ${ }^{29}$, Do Hoon Lim ${ }^{30 *}$, Dong-Sup Chung ${ }^{20 *}$; \\ KSNO Guideline Working Group
}

Received August 20, 2019

Revised August 22, 2019

Accepted September 30, 2019

\section{Correspondence}

Do Hoon Lim

Department of Radiation Oncology,

Samsung Medical Center,

Sungkyunkwan University School of

Medicine, 81 Irwon-ro, Gangnam-gu,

Seoul 06351, Korea

Tel: $+82-2-3410-2600$

Fax: +82-2-3410-2609

E-mail: dh8lim@skku.edu

Dong-Sup Chung

Department of Neurosurgery,

Incheon St. Mary's Hospital,

College of Medicine,

The Catholic University of Korea,

56 Dongsu-ro, Bupyeong-gu,

Incheon 21431, Korea

Tel: $+82-32-280-5841$

Fax: +82-32-280-5991

E-mail: dschung@catholic.ac.kr

*These authors contributed equally to this work as a corresponding author.
Background There was no practical guideline for the management of patients with central nervous system tumor in Korea in the past. Thus, the Korean Society for Neuro-Oncology (KSNO), a multidisciplinary academic society, developed the guideline for glioblastoma successfully and published it in Brain Tumor Research and Treatment, the official journal of KSNO, in April 2019. Recently, the KSNO guideline for World Health Organization (WHO) grade III cerebral glioma in adults has been established.

Methods The Working Group was composed of 35 multidisciplinary medical experts in Korea. References were identified by searches in PubMed, MEDLINE, EMBASE, and Cochrane CENTRAL databases using specific and sensitive keywords as well as combinations of keywords. Scope of the disease was confined to cerebral anaplastic astrocytoma and oligodendroglioma in adults.

Results Whenever radiological feature suggests high grade glioma, maximal safe resection if feasible is globally recommended. After molecular and histological examinations, patients with anaplastic astrocytoma, isocitrate dehydrogenase $(I D H)$-mutant should be primary treated by standard brain radiotherapy and adjuvant temozolomide chemotherapy whereas those with anaplastic astrocytoma, NOS, and anaplastic astrocytoma, IDH-wildtype should be treated following the protocol for glioblastomas. In terms of anaplastic oligodendroglioma, IDH-mutant and 1p19q-codeletion, and anaplastic oligodendroglioma, NOS should be primary treated by standard brain radiotherapy and neoadjuvant or adjuvant PCV (procarbazine, lomustine, and vincristine) combination chemotherapy.

Conclusion The KSNO's guideline recommends that WHO grade III cerebral glioma of adults should be treated by maximal safe resection if feasible, followed by radiotherapy and/or chemotherapy according to molecular and histological features of tumors.

Key Words Korean Society for Neuro Oncology; Guideline; Grade III Gliomas; Practice.

\section{INTRODUCTION}

Historically, the 2007 World Health Organization (WHO) classification of central nervous system (CNS) tumours classified anaplastic astrocytoma, anaplastic oligodendroglioma,

This is an Open Access article distributed under the terms of the Creative Commons Attribution Non-Commercial License (https://creativecommons.org/licenses/by-nc/4.0) which permits unrestricted non-commercial use, distribution, and reproduction in any medium, provided the original work is properly cited.

Copyright $\odot 2019$ The Korean Brain Tumor Society, The Korean Society for NeuroOncology, and The Korean Society for Pediatric Neuro-Oncology anaplastic oligoastrocytoma, anaplastic ependymoma, and anaplastic pleomorphic xanthoastrocytoma as grade III gliomas. Actually, WHO grade III gliomas are not common as glioblastomas. All other malignant gliomas including WHO grade II and III gliomas are composed of $10.2 \%$ of all primary CNS tumors in the United State [1] and 5.3\% in Korea [2]. Such classification was based on morphological features on light microscopy of hematoxylin and eosin-stained sections, immunohistochemical expression of lineage associated proteins, and ultrastructural characterization [3]. However, stud- 
ies over the past two decades have clarified the genetic basis of tumorigenesis for some brain tumor entities, raising the possibility that such an understanding may contribute to new classification of these tumors [4]. The use of integrated phenotypic and genotypic parameters for classification of gliomas adds a level of objectivity that has been missing from some aspects of the diagnostic process in the past [5]. According to the new WHO classification of CNS tumours published in 2016, the following gliomas are classified as WHO grade III gliomas: 1) anaplastic astrocytoma, isocitrate dehydrogenase (IDH)-mutant, 2) anaplastic oligodendroglioma, IDH-mutant and 1p19q-codeleted, 3) anaplastic pleomorphic xanthoastrocytoma, 4) ependymoma, v-rel avian reticuloendotheliosis viral oncogene homolog $\mathrm{A}$ (RELA) fusion-positive, and 5) anaplastic ependymoma [4].

In terms of genotypic characteristics, mutation status of IDH1/2 and codeletion of $1 \mathrm{p} 19 \mathrm{q}$ are more important to diagnose WHO grade III gliomas than methylation status of the O6-methyl guanine DNA methyltransferase (MGMT) gene promoter. Several WHO grade III gliomas in recent classification have morphological characteristics of those in previous pathological classification. If they do not have IDH-mutation, they have a behavior of WHO grade IV gliomas. Therefore, although these gliomas are not categorized as grade III, they should be treated with the same protocol for WHO grade IV gliomas [4].

Despite WHO grade III gliomas have better outcome and longer survival than WHO grade IV gliomas, results of treatment are still very disappointing. There are certain obstacles to overcome these tumors because of frequent recurrence, common progression to higher grade gliomas, and refractoriness to conventional therapies, etc. [6]. Therefore, many clinical trials have been performed and many are also ongoing to overcome these serious diseases. Although there are no new therapeutic modalities such as those for WHO grade IV gliomas, combinations of conventional cytotoxic chemotherapy agents and other agents such as target therapy and/ or immunotherapy have been developed [7-9]. To apply recently established combinations or newly developed agents for patients with grade III gliomas, comprehensive and advanced cancer networks or academic societies for CNS tumors of western countries such as the National Comprehensive Cancer Network (NCCN) [10-12] and the European Association for Neuro-Oncology (EANO) [13] are updating their guidelines for CNS tumor regularly. Besides clinical characteristics such as age of patients, adjuvant temozolomide, and methylation status of MGMT gene promoter as prognostic factors for patients with WHO grade III gliomas $[7,8]$, genetic background such as IDH mutation and codeletion of $1 \mathrm{p} 19 \mathrm{q}$ are also important to estimate the prognosis of patients with WHO grade III gliomas [6].

However, it is a critical point that circumferences of treating patients with WHO grade III gliomas in Korea are much different from those in other countries. The incidence of WHO grade III gliomas is relatively lower [2] and clinical practice is much limited in Korea because Korean government supports medical care financially by the National Health Insurance System which limits application of updated therapeutic modalities to patients.

Although the clinical practical guideline for WHO grade IV gliomas has been just developed and published recently by the Korean Society for Neuro-Oncology (KSNO), a multidisciplinary academic society for CNS tumors [14], the actual guideline for the management of patients with other CNS tumors in Korea is not available yet. The KSNO established a Working Group for developing practice guideline for CNS tumor in February 2018. Recently it is developing a guideline for CNS tumor based on updated information. The objective of KSNO guideline is to provide physicians with evidencebased recommendations and consensus expert opinion for managing patients with glioblastomas in daily clinical practice. It will also serve as a source of knowledge for institutions and insurance companies involved in cancer care in Korea.

\section{KSNO GUIDELINE WORKING GROUP}

A Working Group was appointed by the KSNO in February 2018 to establish guidelines on the management of glioblastoma patients. These guidelines should be optimized considering the unique medical circumstance in Korea. The KSNO Guideline Working Group was composed of 35 medical experts in Korea, including 18 neurosurgeons, 8 radiation oncologists, 1 medical oncologist, 2 neuroradiologists, 3 pediatric oncologists, 2 pathologists, and 1 neurologist. As there is no medical specialty for neuro-oncology in Korea, neurosurgeons (especially brain tumor surgeons) usually play the role of neuro-oncologists in clinical practice.

References were identified by searching PubMed, MEDLINE, EMBASE, and Cochrane CENTRAL databases using specific and sensitive keywords as well as combinations of keywords. Scope of the disease was confined to cerebral anaplastic astrocytoma and anaplastic oligodendroglioma in adults aged $\geq 18$ years because other WHO grade III gliomas are relatively uncommon in Korea. Therefore, WHO grade III glioma in the spinal cord, anaplastic ependymoma, or anaplastic pleomorphic xanthoastrocytoma in the brain was not included. Abstracts presented at the official year-end conference of KSNO in December 2018 were considered relevant. When available, we also collected existing guidelines from national multidisciplinary neuro-oncological societies such as 


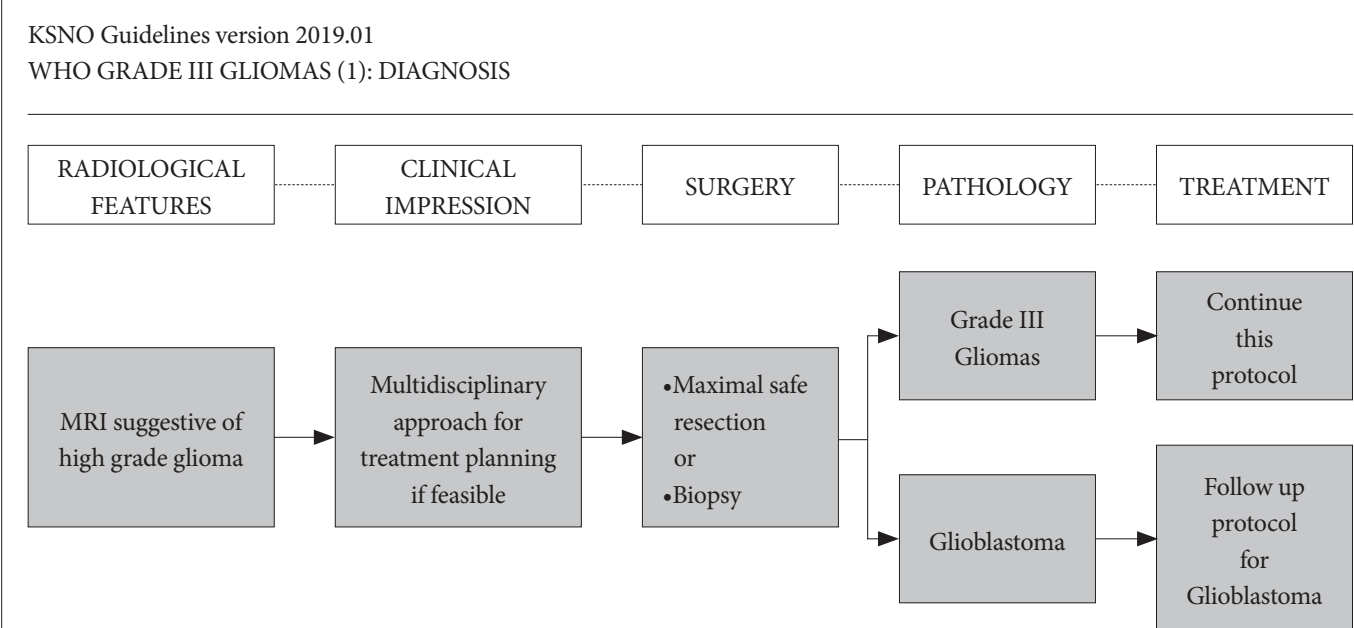

Fig. 1. Guideline for diagnosis of WHO grade III gliomas. To diagnose WHO grade III gliomas, MRI with gadolinium enhancement is essential. A multidisciplinary approach for treatment planning is recommended if feasible. Primarily, WHO grade III gliomas should be diagnosed histopathologically for tissues obtained by neurosurgical intervention. KSNO, Korean Society for Neuro-Oncology; MRI, magnetic resonance image; WHO, World Health Organization.

the NCCN and EANO. The final reference list was generated based on originality and relevance to the scope of this guideline. The strategy of establishing this guideline was mainly based on NCCN and EANO guidelines with modifications and changes according to the unique background of Korea.

Scientific evidence was assessed and graded according to the following categories: high level evidence (evaluated from multiple populations and derived from randomized clinical trials or meta-analysis or systemic review) and low level evidence (evaluated from limited population and derived from non-randomized studies, including observational studies, cohort studies, and case-control studies).

To establish recommendation levels, the following criteria were used. Level I (strong recommendation) required a high level of evidence and uniform consensus among panels. Level II (weak recommendation) required a high level of evidence but not uniform consensus among panels or a low level of evidence but uniform consensus among panels. Level III (individual decision) required a low level of evidence but not uniform consensus among panels. Level IV (not recommendable) required contents being not beneficial or harmful. Recommendations with level I or level II evidence were not marked. However, those with level III and level IV evidence were marked at the end of each recommendation in this guideline.

\section{DIAGNOSIS OF WHO GRADE III GLIOMAS}

When radiological feature suggests a high-grade glioma, multidisciplinary approach for treatment planning should be considered if feasible (Fig. 1). Computed tomography (CT) is insufficient to suggest a high-grade glioma even with contrast enhancement. Therefore, magnetic resonance image (MRI) with contrast enhancement is essential to diagnose a highgrade glioma. In order to obtain sufficient tissue for histopathological diagnosis, neurosurgical intervention is mandatory even if it is for stereotactic biopsy. To achieve maximal safe resection, neuronavigation system, intraoperative CT or MRI, intraoperative ultrasonography, intraoperative mapping technique, and fluorescence-guidance with 5-aminolevuliniv acid are recommended. Histopathological diagnosis should be officially based on 2016 WHO Classification of Tumors of the Central Nervous System [4]. Codeletion of 1p19q testing and IDH1/IDH2 mutation test are essential parts of molecular diagnosis for grade III gliomas. Also, MGMT promoter methylation test is required for workup of WHO grade III gliomas. If the histopathological diagnosis is WHO grade IV gliomas, patients should be treated based on guideline for WHO grade IV gliomas instead of this guideline [14].

\section{ADJUVANT TREATMENT OF WHO GRADE III GLIOMAS}

\section{Anaplastic astrocytoma, $I D H$-mutant}

In terms of histopathological and molecular features, the following modalities should be considered for patients with anaplastic astrocytoma, $I D H$-mutant (Fig. 2): standard brain radiotherapy and adjuvant temozolomide chemotherapy, or standard brain radiotherapy with concurrent and adjuvant temozolomide chemotherapy, or standard brain radiotherapy with neoadjuvant or adjuvant PCV (procarbazine+lomustine +vincristine) combination chemotherapy, or standard brain 
KSNO Guidelines version 2019.01

WHO GRADE III GLIOMAS (2): ADJUVANT THERAPY (1)

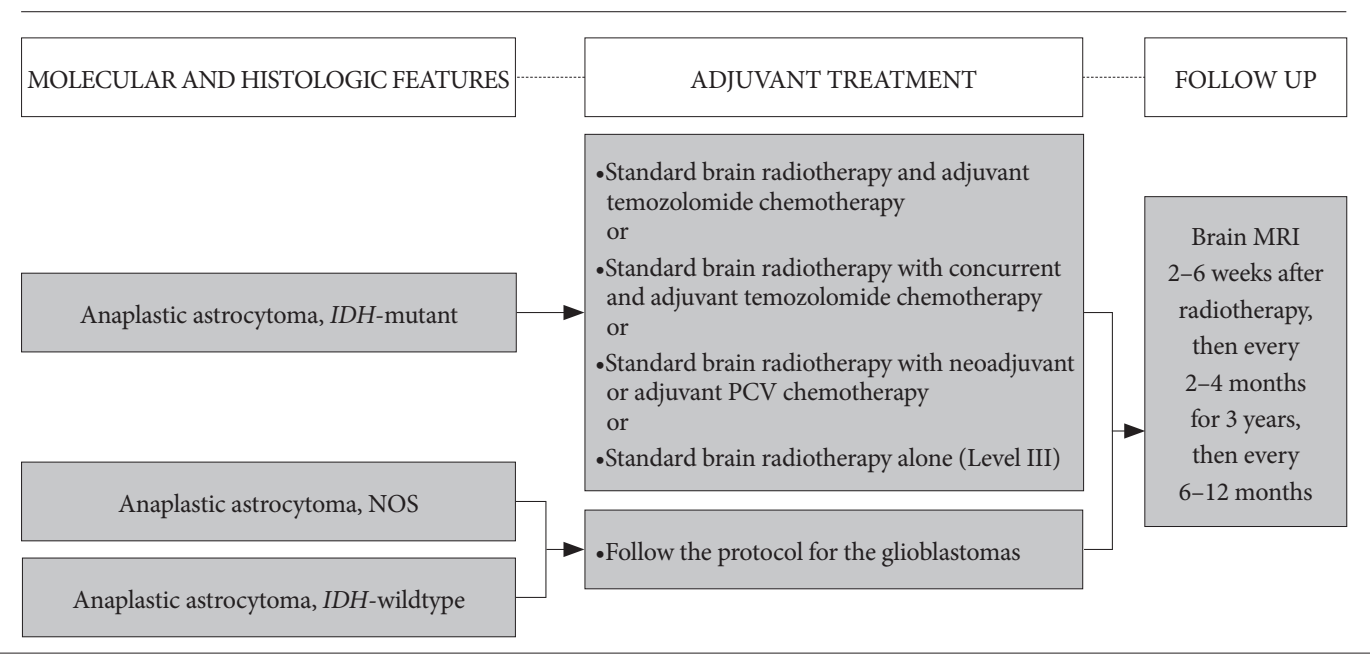

Fig. 2. Guideline for adjuvant therapy of patients with anaplastic astrocytoma, IDH-mutant, NOS, and IDH-wildtype. 1) For the patients with anaplastic astrocytoma, $I D H$-mutant, standard brain radiotherapy and adjuvant temozolomide chemotherapy, or standard brain radiotherapy with concurrent and adjuvant temozolomide chemotherapy, or standard brain radiotherapy with neoadjuvant or adjuvant PCV chemotherapy, or standard brain radiotherapy alone are recommended. 2) For the patients with anaplastic astrocytoma, NOS, and IDH-wildtype, the practical protocol for glioblastoma patients is recommended. IDH, isocitrate dehydrogenase; KSNO, Korean Society for Neuro-Oncology; PCV, procarbazine+lomustine+vincristine; MRI, magnetic resonance image.

radiotherapy alone (Level III). Adjuvant temozolomide is recommended at $150-200 \mathrm{mg} / \mathrm{m}^{2}$ for five consequential days every 4 weeks. The number of cycles of adjuvant temozolomide chemotherapy can be up to 12 (Level III). During concurrent chemoradiotherapy with temozolomide, the dose of temozolomide is recommended at $75 \mathrm{mg} / \mathrm{m}^{2}$. Additional temozolomide after concurrent chemoradiotherapy is recommended at $150-200 \mathrm{mg} / \mathrm{m}^{2}$ for five consequential days every 4 weeks for adjuvant purpose. The number of cycles of adjuvant temozolomide chemotherapy is also up to 12 . The maximum number of cycles of adjuvant chemotherapy using PCV regimen is 6. Despite low evidence, panels have consensus (although not uniform) that standard brain radiotherapy without adjuvant chemotherapy should be considered for WHO grade III glioma patients with poor performance status [Karnofsky Performance Scale (KPS) $\leq 60$ ] (Level III).

\section{Anaplastic astrocytoma, NOS, and anaplastic astrocytoma, $\mathrm{IDH}$-wild type}

As most anaplastic astrocytomas (except genetic feature of $I D H$-mutant) usually have similar clinical and pathological behaviors, patients should be treated based on the guideline for grade IV gliomas (Fig. 2).

\section{Anaplastic oligodendroglioma}

According to the 2016 WHO classification of tumors of the CNS, prior diagnoses of oligoastrocytoma and anaplastic oligoastrocytoma are newly designated as NOS categories since these diagnoses should be rendered only in the absence of diagnostic molecular testing or in the very rare instance of a dual genotype oligoastrocytoma [4]. Therefore, the following treatment should be considered for anaplastic oligodendrogliomas including those with $\mathrm{IDH}$-mutant and 1p19q-codeletion and those without result of molecular testing: standard brain radiotherapy and neoadjuvant or adjuvant PCV combination chemotherapy, or standard brain radiotherapy with concurrent and adjuvant temozolomide chemotherapy, or standard brain radiotherapy alone (Level III) (Fig. 3). The maximum number of cycles of the adjuvant chemotherapy using PCV regimen is 6. During concurrent chemoradiotherapy with temozolomide, the dose of temozolomide is recommended at $75 \mathrm{mg} / \mathrm{m}^{2}$. Additional temozolomide after concurrent chemoradiotherapy is recommended at $150-200 \mathrm{mg} / \mathrm{m}^{2}$ for five consequential days every 4 weeks for adjuvant purpose. The number of cycles of the adjuvant temozolomide chemotherapy is also up to 12. Despite low evidence, the panels have consensus (although not uniform) that standard brain radiotherapy without adjuvant chemotherapy for WHO grade III glioma should be considered for patients with poor performance status (KPS $\leq 60$ ) (Level III).

\section{Follow up radiological study}

Radiological follow-up using MRI with gadolinium enhancement is recommended. For patients with anaplastic astrocytoma, regular check-up at 2-6 weeks after radiotherapy, then every $2-4$ months for 3 years, then every 6-12 months is 
KSNO Guidelines version 2019.01

WHO GRADE III GLIOMAS (2): ADJUVANT THERAPY (2)

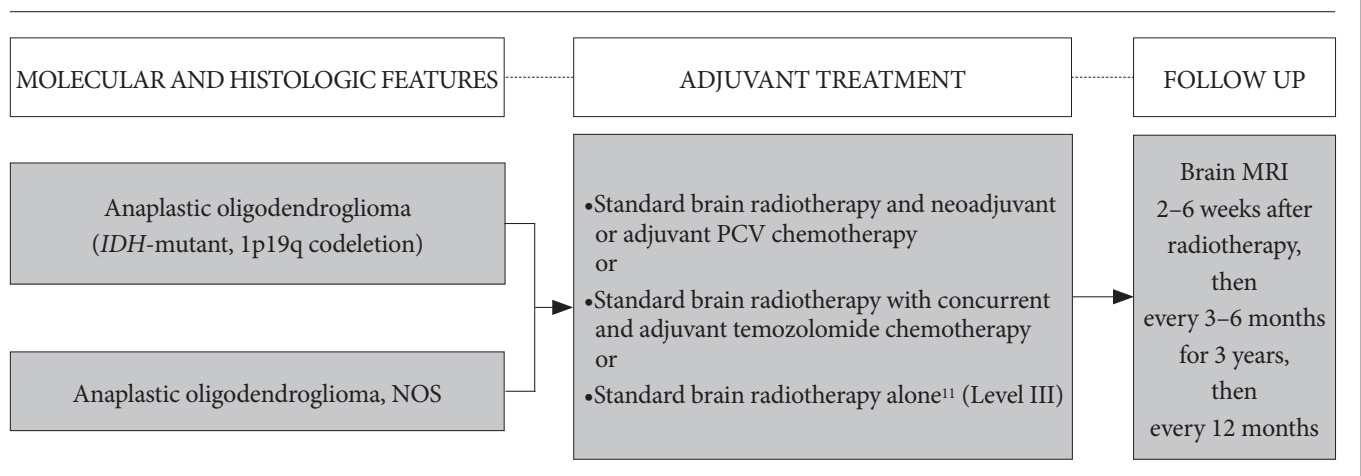

Fig. 3. Guideline for adjuvant therapy of patients with anaplastic oligodendroglioma. The patients should be treated with following modalities; 1) standard brain radiotherapy and neoadjuvant or adjuvant PCV chemotherapy, or 2) standard brain radiotherapy with concurrent and adjuvant temozolomide chemotherapy, or 3) standard brain radiotherapy alone. KSNO, Korean Society for Neuro-Oncology; PCV, procarba zine+lomustine+vincristine; MRI, magnetic resonance image.

recommended (Fig. 2). For patients with anaplastic oligodendrogliomas, regular check-up 2-6 weeks after radiotherapy, then every 3-6 months for 3 years, then every 12 months (Fig. 3 ) is recommended regardless of age or therapeutic option. In spite of the absence of definite evidence for interval of followup imaging study, there is a uniform consensus among the panels for the above schedule for follow-up radiologic study.

MRI at this time can be much informative to detect radiation-induced brain swelling and pseudoprogression after radiotherapy. The physician can care patients more comfortably based on appropriate imaging study.

\section{TREATMENT OF RECURRENT WHO GRDE III GLIOMAS}

Diagnosis of WHO grade III glioma's recurrence can be indistinguishable from pseudoprogression on MRI within the first 3 months after completion of radiotherapy. However, the following radiologic findings can suggest recurrence as recommended by radiologic assessment of neurooncology (RANO) criteria [15]: 1) 25\% or more increase in enhancing lesions despite stable or increasing steroid dose, 2) significant increase of the lesion in fluid-attenuated inversion recovery (FLAIR) image and T2 weighted image not attributable to other non-tumor causes, and 3) any new lesion. Also, if clinical deterioration (not attributable to other non-tumor causes or steroid decrease) occurs simultaneously, true progression is strongly suggested. If recurrence is suspected in conventional MRI, it is better to consider the following options: 1) undergoing biopsy, 2) checking functional radiologic study such as MR spectroscopy, MR perfusion, brain positron emission tomography (PET)-CT, or 3) checking MRI again and comparing changes that may be due to progression versus radiation necrosis. When recurrence of the tumor is suggested clinically and radiologically, surgical resection is always recommended, if feasible. Even in case of recurrence of WHO grade III gliomas with difficulty for complete resection such as diffuse, multi-focal, or deep located lesion, surgical treatment can be considered to reduce mass effect, improve neurological symptoms, and define progression into WHO grade IV gliomas. After confirm recurrence, the following salvage treatment should be considered: systemic chemotherapy and/or reirradiation and/or clinical trials and/or supportive treatment if patient has poor performance status (Fig. 4).

The following adjuvant systemic therapies can be considered for recurrent WHO grade III gliomas based on physician's decision: cytotoxic chemotherapy, target therapy, and/or immunotherapy, etc. However, the efficacy of standard-of-care treatment such as adjuvant temozolomide chemotherapy for recurrent WHO grade III glioma is suboptimal for salvage purpose. Thus, for eligible patients, consideration of clinical trials is highly encouraged.

\section{PRINCIPLES OF MANAGEMENT FOR WHO GRADE III GLIOMAS}

\section{Brain imaging}

Many imaging modalities are available and used in neurooncology primarily to make treatment decisions in Korea. Imaging is always recommended to investigate emergent signs or symptoms. MRI of the brain (with and without contrast) is the gold standard modality to investigate brain tumors. It provides a static picture of brain tumors. It has a benefit in that it provides a reasonably good delineation of tumors. In MRI, high grade tumors and brain leptomeningeal metastases usually show enhancement while low-grade tumors usually do 
KSNO Guidelines version 2019.01

WHO GRADE III GLIOMAS (3): RECURRENT DISEASE

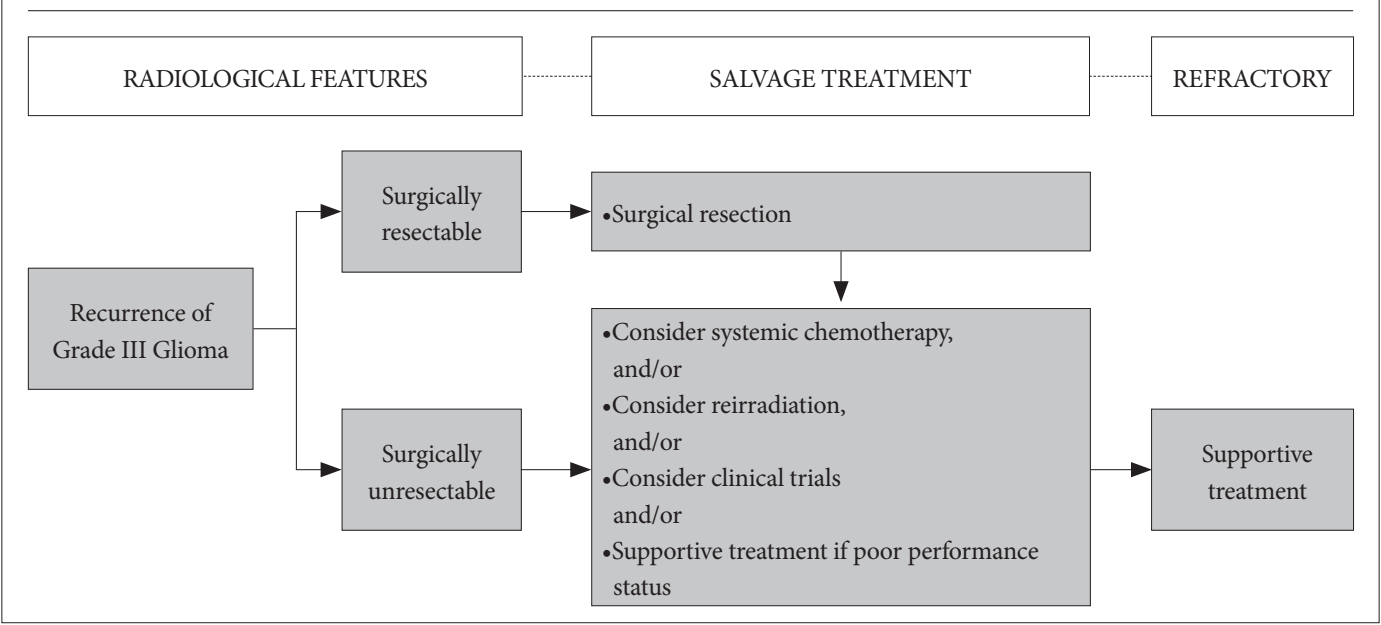

Fig.4. Guideline for recurrent WHO grade III gliomas. Surgical resection is always recommended if feasible. After surgical resection, the following therapeutic options are considered: 1) systemic chemotherapy, and/or 2) reirradiation, and/or 3) enrollment of clinical trials and/or 4) supportive treatment if poor performance status. KSNO, Korean Society for Neuro-Oncology.

not. However, MRI has a limitation in that it is sensitive to movement. In addition, metallic objects can cause artifacts. Thus, patients with implantable devices cannot receive an MRI. Moreover, claustrophobia or renal insufficiency may be an issue. Postoperative brain MRI should be performed within 24-72 hours after surgery for gliomas and other tumors to determine the extent of resection.

CT scan of the brain (with and without contrast) is usually considered for patients who cannot undergo an MRI. CT has a benefit of avoiding claustrophobia or implanted devices in the body. It is faster than an MRI. However, CT has a limitation in that it lacks resolution, especially for tumor located within the posterior fossa and for patients with renal insufficiency.

MR spectroscopy can be used to assess metabolites within tumor and normal tissues. It may be useful for differentiating tumors from radiation necrosis. It may also be helpful for grading tumors or assessing therapeutic response. The area showing the most abnormal features would be the best place to target for a biopsy. However, it has limitation for tumors near vessels, air space, or bone.

MR perfusion can be used to measure cerebral blood volume in tumors. It may be useful for differentiating the grade of tumor or tumor versus radiation necrosis. The area with the highest perfusion would be the best place for a biopsy. However, it has also limitation for tumors near vessels, air space, or bone, and small-volume lesions.

PET-CT using fluorodeoxyglucose (FDG) has a limitation for functional diagnosis of WHO grade III glioma due to high uptake of FDG in the brain with normal biologic metabolism. However, PET-CT using methionine, an essential amino acid, may be helpful for differentiating the tumor progression versus radiation necrosis. This technology is not commonly used in the clinical field in Korea. Further development of application is necessary for casual use in clinical practice.

\section{Multidisciplinary care}

During treatment, most patients with WHO grade III gliomas will be managed by various subspecialists. Close and regular communication among all providers across multi-disciplines is essential. Utilization of a brain tumor board or multidisciplinary clinic care models can facilitate interactions among various subspecialists, ideally including allied health services (e.g., physical, occupational and speech therapies, nursing, psychology, and social services) to optimize treatment plan recommendations.

As treatment proceeds, it is important that the patient and his/her family understand the role of each team member. One attending physician who mainly cares for the patient should be determined as early as possible and the attending physician should contact patients regularly for follow up. Additionally, the attending physician can facilitate referral to appropriate specialist.

The patient is strongly encouraged to participate in various clinical trials. Practitioners should discuss any local, regional, and national options for which the patient may be eligible and advantages/disadvantages of participation. The center that treats neuro-oncology patients should encourage patients to participate in large collaborative trials in order to have another option for patients.

Throughout treatment, patient's health-related quality of 
life should remain the highest priority and guide clinical decision-making. While therapeutic response on radiologic study can be a good indicator of successful therapy, other non-radiologic indicators of therapeutic response such as overall well-being, cognitive function, physical and motor functioning in day-to-day activities, communication ability, social functioning and family interactions, nutrition, pain control, long-term consequences of treatment, and psychological issues should also be considered.

Patients should be informed of the possibility of pseudoprogression, its approximate incidence, and potential investigations that may be needed if pseudoprogression is suspected. Close follow-up imaging, MR spectroscopy, PET-CT imaging, and repeat neurosurgical intervention including biopsy and surgical resection may be necessary if clinically indicated.

\section{Brain tumor surgery}

General principles of surgical resection of WHO grade III glioma of brain are as follows: gross total resection when appropriate, minimal surgical morbidity, and accurate diagnosis. The following factors should be considered when deciding surgical resection: age, performance status, feasibility of decreasing the mass effect with surgery, resectability, including number of lesions, location of lesions, time since last surgery in recurrent patients, and new versus recurrent tumor [12]. Suspected pathology should also be considered with the following points: benign versus malignant, possibility of other non-cancer diagnoses, and projected natural history. For patients with $I D H 1$ mutants, there is evidence suggesting that a supra-marginal resection is the most appropriate, which would include not only enhancing areas, but also T2/FLAIR areas when appropriate in terms of safe surgical approach with the use of any and all surgical adjuncts possible [16].

Options of surgical resection include gross total resection where feasible, stereotactic biopsy, and open biopsy/debulking followed by planned observation or adjuvant therapy. In order to obtain the maximal safe resection, neuronavigation systems, intraoperative MRI or CT, intraoperative ultrasonography, fluorescence-guided with 5-aminolevulinic acid, and intraoperative mapping techniques may be helpful.

For histopathological diagnosis and genetic information, sufficient tissue should be sent to the pathologist for neuropathology evaluation and molecular correlates analysis. Frozen section analysis when possible can help with intraoperative decision making. The tissue should be reviewed by an experienced neuropathologist.

Postoperative brain MRI should be performed within 2472 hours after surgery for WHO grade III glioma to determine the extent of resection. The extent of resection should be judged based on postoperative imaging study. It should be used as a baseline to assess further therapeutic efficacy or tumor progression.

\section{Pathology examination}

Incorporation of relevant diagnostic markers, including histopathologic and molecular information described in the 2016 WHO Classification of Tumors of the Central Nervous System, should be considered as standard practice for tumor classification. Molecular/genetic characterization complements standard histologic analysis. It provides additional diagnostic and prognostic information that can greatly improve diagnostic accuracy, influence treatment selection, and possibly improve management decision-making.

For standard histopathologic examination of WHO grade III glioma of brain, basic histologic examination is performed based on the description in the WHO Classification of Tumors of the Central Nervous System [4]. Interobserver discrepancies in histologic diagnosis and grading are recognized issues due to the inherently subjective nature of certain aspects of histopathologic interpretation (e.g., astrocytic vs. oligodendroglial morphology). In addition, surgical sampling does not always capture all relevant diagnostic features of morphologically heterogeneous tumors.

Through genetic and molecular testing, WHO grade III gliomas can be differentiated more accurately in terms of prognosis and, in some instances, response to different therapies. However, molecular/genetic characterization does not replace standard histologic assessment. It serves as a complementary approach to provide additional diagnostic and prognostic information that often enhances treatment selection. Although there are no identified targeted agents with demonstrated efficacy in WHO grade III glioma, the panel encourages molecular testing of tumors such as next generation sequencing because if a driver mutation is detected, it may be reasonable to treat patient with a targeted therapy on a compassionate use basis, and/or the patient may have more treatment options in the context of a clinical trial.

Molecular testing also has a valuable role in improving diagnostic accuracy and prognostic stratification that may inform treatment selection. For example, codeletion of $1 \mathrm{p} 19 \mathrm{q}$ testing is an essential part of molecular diagnostics for oligodendrogliomas. Therefore, $1 \mathrm{p} 19 \mathrm{q}$ codeletion testing should be considered to differentiate astrocytoma from oligodendrogliomas. Also, IDH1/IDH2 mutation testing is required for differential diagnosis between $I D H$-mutant and $I D H$-wildtype tumors. Additionally, MGMT promoter methylation is an essential part of molecular diagnostics for all high-grade gliomas.

\section{Radiotherapy}

Optimal timing of radiotherapy after surgical resection is 
not established in WHO grade III glioma of brain. Delay in radiotherapy has not been shown to decrease survival. However, we recommend radiotherapy to be initiated at postoperative 2-6 weeks after full recovery from surgical interventions. Whenever radiotherapy is planned for patients with WHO grade III gliomas, pre- and post-operative MRIs should be performed to define all target volumes, including gross and clinical tumor volume (GTV and CTV). Additional MRI at the time of radiotherapy simulation can be used to account for changes in surgical cavity or lesions. CT-based 3-dimensional calculation of dose distribution should be performed at any circumstance.

The GTV should encompass preoperative tumor bed and enhancing lesions on $\mathrm{T} 1$-weighted image of postoperative MRI. The preoperative tumor bed should not be directly delineated on the registered preoperative MRI on the planning CT in patients undergoing surgical resection. T2/FLAIR signals can also be included in the GTV if needed. Historical trials and international groups [e.g., Radiation Therapy Oncology Group (RTOG) and European Organization for Research and Treatment of Cancer (EORTC)] generally recommend an expansion of $2-2.5 \mathrm{~cm}$ for CTV delineation to account for subclinical tumor infiltration. However, smaller CTV-margins may be reasonable based on retrospective studies (as low as $0.5 \mathrm{~cm}$ ). Simple expansion from the GTV should be avoided. CTV should always be modified based on anatomical barriers for tumor infiltrations. A margin of 3-5 $\mathrm{mm}$ from the CTV is usually recommended to create the planning target volume to account for errors from imageregistration and daily set-up of patients. However, the margin can also be reduced if daily image-guidance is performed.

A total dose of 59.4-61.2 Gy is recommended as standard radiotherapy using a daily fraction of 1.8-2.0 Gy. A reduced field can be used after delivering 45-50.4 Gy. However, field reduction does not always need to be performed sequentially. When intensity-modulated radiotherapy (IMRT or IMPT) is used to avoid critical organs, simultaneous integrated boost techniques can be used. For fragile patients with old age or poor performance requiring hypofractionated radiotherapy in 1-4 weeks, the following dose-fractionation regimens are frequently adopted: $40.05 \mathrm{~Gy} / 15$ fractions, $34 \mathrm{~Gy} / 10$ fractions, $50 \mathrm{~Gy} / 20$ fractions, and $25 \mathrm{~Gy} / 5$ fractions. No single scheme has been proven to be superior to another. Different schedules can also be used based on physician's decision. The optimal margin for CTV expansion has not been established yet. Therefore, we recommend physicians to follow the principles of standard radiotherapy. Absolute cumulative dose limits or optimal intervals between radiotherapy sessions are not established for these gliomas yet. Thus, delivery, dose, fraction, target volume, and techniques for reirradiation should be decided by a brain tumor radiation oncologist and through a multidisciplinary discussion whenever feasible.

\section{DISCUSSION}

This is the second practical guideline for CNS tumors developed by KSNO Guideline Working Group. Major difference of KSNO guideline between WHO grade IV and III glioma is the way to classify patients. The first step of the guideline approaching patients with WHO grade IV gliomas was based on basic clinical features such as age and performance status of patients. On the contrary, the first step of the guideline approaching those with WHO grade III gliomas was based on histological and molecular features of tumors. The reason is that molecular characteristics such as the status of $I D H 1 / 2$ mutation and codeletion of $1 \mathrm{p} 19 \mathrm{q}$ are the more important ones for predicting therapeutic outcome of WHO grade III glioma patients than clinical characteristics. The best prognosis was found in patients with $I D H 1 / 2$ mutant and $1 \mathrm{p} 19 \mathrm{q}$ codeleted tumors [6].

While histological definition of WHO grade IV glioma is relatively uncontentious among neuropathologists, histologically defined groups of anaplastic astrocytoma, anaplastic oligodendroglioma, and anaplastic oligoastrocytoma remain a major challenge for various reasons: 1) there is poor interobserver agreement when diagnoses and grading are made by histological criteria alone [17], particularly concerning the classification of oligoastrocytomas [18], 2) their clinical courses are highly variable, and 3) clinical management for them remains poorly standardized [19]. Therefore, in the 2016 WHO classification of CNS tumors, prior diagnoses of oligoastrocytoma and anaplastic oligoastrocytoma are now designed as NOS categories since these diagnoses should be rendered only in the absence of diagnostic molecular testing or in the very rare instance of a dual genotype oligoastrocytoma [4].

As mentioned above, almost all medical practices are under regulation by the National Health Insurance System in Korea. Limited therapeutic modalities are included in this guideline because the national system does not cover various therapeutic modalities such as temozolomide alone chemotherapy for newly diagnosed WHO grade III glioma even if patients have poor performance status, thus limiting clinical practice in the field of neuro-oncology in Korea. The Korean government permits only two therapeutic options such as standard brain radiotherapy and/or adjuvant nitrourea-based cytotoxic chemotherapy regardless of molecular features including status of $I D H 1 / 2$ mutation and $1 \mathrm{p} 19 \mathrm{q}$ codeletion. Therefore, this guideline basically divided gliomas according to critical molecular and histological features instead of clini- 
cal status. Although the Korean National Health Insurance System does not permit concurrent chemoradiotherapy using temozolomide for newly diagnosed WHO grade III gliomas, the panels have a uniform consensus that this therapeutic option can be considered for patients with such situation.

Despite this guideline emphasizes the importance of molecular and genetic diagnosis for the categorization of patients, only three tests were included in this guideline: $I D H 1 / 2$ mutation, $1 \mathrm{p} 19 \mathrm{q}$ codeletion, and methylation status of MGMT gene promoter. As mentioned in this guideline, IDH1/2 mutation test is an essential part of molecular diagnostics for WHO grade III glioma because this mutation can distinguish lowergrade glioma from primary glioblastoma [20,21]. IDH mutations are commonly associated with MGMT gene promoter methylation [22], relatively favorable prognosis [23], and survival benefit for patients treated with radiation or alkylating chemotherapy [24]. Wild-type IDH1/IDH2 is associated with increased risk of aggressive disease [22], which makes anaplastic astrocytoma, $I D H$-wildtype follow the guideline for WHO grade IV gliomas in this guideline. In fact, this molecular testing is reliably screened by mutation-specific immunohistochemistry without any practical difficulties in Korea. In the same manner, $1 \mathrm{p} 19 \mathrm{q}$ deletion test is an essential part of molecular diagnostics for WHO grade III glioma, especially oligodendrogliomas. This codeletion confers a favorable prognosis. It is predictive of response to alkylating chemotherapy and combination therapy with radiation and alkylating chemotherapy $[25,26]$. Codeletion of $1 p$ and $19 q$ is generally detected by fluorescence in situ hybridization or polymerase chain reaction in regular practice in Korea. Although the NCCN [12] and EANO guideline [13] recommend more tests for genetic alteration of grade III gliomas, including ATP-dependent Xlinked helicase $(A T R X)$ gene mutation, telomerase reverse transcriptase (TERT) promoter mutation, and H3F3A mutation, these more complex and detailed molecular tests were not included in this guideline due to practical limitations of individual institute in Korea.

Fortunately, the Korean National Health Insurance System allows recurrent WHO grade III glioma to be treated by temozolomide as well as lomustine and PCV combination regimen. However, bevacizumab which is permitted for patients with recurrent grade IV glioma is still limited for those with recurrent grade III glioma. Other recommended regimens in NCCN guideline [12] such as bevacizumab combined with chemotherapeutic agents (lomustine, temozolomide, and carboplatin, etc.) are also unavailable in Korea. Fortunately, recent data from CeTeG/NOA-09 randomized, open-label, phase 3 clinical trial have suggested that lomustine-temozolomide combination chemotherapy might improve survival compared to temozolomide standard therapy in patients with newly di- agnosed glioblastoma and methylated MGMT gene promoter [27]. Although this result was obtained not for WHO grade III glioma patients, but for glioblastoma patients, lomustine which is more commonly used in Korea can be considered for these patients after validation by further studies.

At recurrence, there is no standard or effective treatment for WHO grade III glioma in Korea or western countries. In fact, a recent randomized controlled phase 2 EORTC trial (TAVAREC) using combination chemotherapy of bevacizumab and temozolomide for recurrent WHO grade II and III gliomas without $1 \mathrm{p} 19 \mathrm{q}$ codeletion failed to show improved overall survival [7]. For recurrent glioblastomas, a multicenter, open label, randomized, controlled, phase 2 trial (REGOMA) has shown survival benefit of regorafenib which is a target agent for vascular endothelial growth factor (VEGF)-independent angiogenesis [28]. This drug can be expected to be a new potential treatment for patients with glioblastoma as well as WHO grade III gliomas after investigation in an adequately powered phase 3 study. Therefore, much less regimens can be used for patients, including several alkylating agents in Korea, than those in other countries. Due to a smaller number of patients in Korea, global pharmaceutical companies are reluctant to enroll Korean patients into their clinical trials for new drugs such as immunotherapeutic agents. Therefore, chances to participate in clinical trials in Korea are very small. This background results in clinical trials to be recommended only at the time of recurrence in this guideline as in KSNO guideline for glioblastoma patients.

The weakness of this KSNO guideline for WHO grade III cerebral gliomas is not different from that of KSNO guideline for glioblastoma. The major weakness of this guideline is limited application to Korean patients with WHO grade III gliomas due to the unique medical atmosphere of Korea. Therefore, it is less helpful for physicians treating patients outside of Korea. In order to use this guideline globally, Asian countries including Japan and China should have a comprehensive network for brain tumor management that can cooperate and share their guidelines. Further, it will be helpful for Asian countries to establish a global guideline that is commonly applicable to these countries. The next hurdle to be overcome is molecular and genetic test for WHO grade III gliomas and whole brain tumors. Genetic information is now rapidly changing, making it difficult to establish definite guideline for clinical practice. The Consortium to Inform Molecular and Practical Approaches to CNS Tumor Taxonomy (cIMPACTNOW) is the notable example that shows rapid change in molecular diagnosis of brain tumors [29,30]. Recently, exome sequencing of anaplastic astrocytomas has suggested a distinct mutation profile from primary glioblastomas, including frequent mutations in Notch pathway genes [31]. In addition, 
this guideline does not include medical management. In clinical practice, physicians are still struggling to manage the mass effect, brain edema, radiation necrosis, seizures, endocrine dysfunctions, fatigues, psychological disorders such as depression and anxiety, and venous thromboembolism [12]. However, the consensus for steroid therapy, the use of antiepileptic drugs, and antipsychotic drugs for these patients is not satisfactory. Finally, this guideline did not include all WHO grade III cerebral gliomas. Only patients with anaplastic astrocytoma and anaplastic oligodendroglioma were subjects for this guideline. Other WHO grade III gliomas such as anaplastic ependymoma or anaplastic pleomorphic xanthoastrocytoma were not included in this guideline. Gliomas of the spinal cord were not subjects of this guideline either. Therefore, the KSNO's Guideline Working Group has plans to continue the process of updating guidelines so that the weakness of this version can be improved.

\section{CONCLUSIONS}

As there was no practical guideline for the management of brain tumor patients, the KSNO developed this guideline that should be applicable for physicians under unique medical circumstances in Korea. The KSNO Guideline Working Group composed of 35 multidisciplinary medical experts in Korea prepared "The KSNO guideline for WHO grade III cerebral glioma in adult: version 2019.01" as the second guideline following the KSNO Guideline for Glioblastoma.

In summary, WHO grade III cerebral gliomas in the adults should be treated by maximal safe resection if feasible followed by radiotherapy and/or chemotherapy according to individual molecular and histopathological features of tumors. As data emerging in the past few years have led to significant changes in the diagnosis, categorization, and treatment of WHO grade III gliomas, we plan to update this guideline consistently. Also, consecutive guideline for other brain tumors such as WHO grade II gliomas, brain metastasis, and meningiomas will be published by the KSNO Guideline Working Group.

\section{Conflicts of Interest}

The authors have no potential conflicts of interest.

\section{Acknowledgments}

This protocol can be applied to adult patients with WHO grade III gliomas of the brain. We specially appreciate the following individuals for their help: Chan Woo Wee, M.D. (Department of Radiation Oncology, Seoul National University Bundang Hospital) for description of the principle of radiotherapy, Soon-Tae Lee, M.D. (Department of Neurology, Seoul National University Hospital) for description of the principle of multidisciplinary care, Jaejoon Lim, M.D. (Department of Neurosurgery, Bundang CHA Medical Center) for systemic review of chemotherapy for WHO grade III gliomas, Kyoung Su Sung, M.D. (Department of Neurosurgery, Dong-A
University Hospital) for systemic review of surgical treatment for WHO grade III gliomas, Jihae Lee, M.D. (Department of Radiation Oncology, Ewha Women's University Mokdong Hospital) for systemic review of radiation therapy for WHO grade III gliomas, and Hyuk-Jin Oh, M.D. (Department of Neurosurgery, Soonchunhyang University Cheonan Hospital) for systemic review of treatment for recurrent WHO grade III gliomas.

\section{Author Affiliations}

${ }^{1}$ Division of Neurooncology and Department of Neurosurgery, Samsung Changwon Hospital, Sungkyunkwan University School of Medicine, Changwon, Korea; ${ }^{2}$ Department of Neurosurgery, Seoul National University Bundang Hospital, Seoul National University College of Medicine, Seongnam, Korea; ${ }^{3}$ Department of Neurosurgery, Bundang CHA Medical Center, CHA University, Seongnam, Korea; ${ }^{4}$ Department of Neurosurgery, Dong-A University Hospital, Dong-A University College of Medicine, Busan, Korea; ${ }^{5}$ Department of Radiation Oncology, Ewha Women's University Mokdong Hospital, Ewha Women's University School of Medicine, Seoul, Korea; ${ }^{6}$ Department of Neurosurgery, Soonchunhyang University Cheonan Hospital, Soonchunhyang University College of Medicine, Cheonan, Korea; ${ }^{7}$ Department of Neurosurgery, Severance Hospital, Yonsei University College of Medicine, Seoul, Korea; ${ }^{8}$ Department of Neurosurgery, Korea University Anam Hospital, Korea University College of Medicine, Seoul, Korea; ${ }^{9}$ Department of Neurosurgery, Samsung Medical Center, Sungkyunkwan University School of Medicine, Seoul, Korea; ${ }^{10}$ Department of Radiation Oncology, St. Vincent's Hospital, College of Medicine, The Catholic University of Korea, Seoul, Korea; ${ }^{11}$ Department of Neurosurgery, Ajou University Hospital, Ajou University School of Medicine, Suwon, Korea; ${ }^{12}$ Department of Pathology, Severance Hospital, Yonsei University College of Medicine, Seoul, Korea; ${ }^{13}$ Division of Medical Oncology, Department of Internal Medicine, Seoul National University Bundang Hospital, Seoul National University College of Medicine, Seongnam, Korea; ${ }^{14}$ Department of Radiation Oncology, Seoul National University Bundang Hospital, Seoul National University College of Medicine, Seongnam, Korea; ${ }^{15}$ Department of Radiology and Research Institute of Radiology, University of Ulsan College of Medicine, Asan Medical Center, Seoul, Korea; ${ }^{16}$ Department of Neurosurgery, Seoul St. Mary's Hospital, College of Medicine, The Catholic University of Korea, Seoul, Korea; ${ }^{17}$ Clinic of Pediatric Oncology, National Cancer Center, Goyang, Korea; ${ }^{18}$ Department of Neurosurgery, Konkuk University Medical Center, Konkuk University School of Medicine, Seoul, Korea; ${ }^{19}$ Department of Neurosurgery, St. Vincent's Hospital, College of Medicine, The Catholic University of Korea, Seoul, Korea; ${ }^{20}$ Department of Neurosurgery, Incheon St. Mary's Hospital, College of Medicine, The Catholic University of Korea, Incheon, Korea; ${ }^{21}$ Department of Radiation Oncology, Yonsei Cancer Center, Yonsei University College of Medicine, Seoul, Korea; ${ }^{22}$ Department of Neurology, Seoul National University Hospital, Seoul National University College of Medicine, Seoul, Korea; ${ }^{23}$ Department of Radiation Oncology, Seoul St. Mary's Hospital, College of Medicine, The Catholic University of Korea, Seoul, Korea; ${ }^{24}$ Department of Pathology, Seoul St. Marry's Hospital, College of Medicine, The Catholic University of Korea, Seoul, Korea; ${ }^{25}$ Department of Neurosurgery, Chonnam National University Hwasun Hospital, Chonnam National University Medical School, Gwangju, Korea; ${ }^{26}$ Department of Pediatrics, Kangbuk Samsung Hospital, Sungkyunkwan University School of Medicine, Seoul, Korea; ${ }^{27}$ Department of Radiology, Seoul National University Hospital, Seoul National University College of Medicine, Seoul, Korea; ${ }^{8}$ Department of Pediatrics, Seoul National University Bundang Hospital, Seoul National University College of Medicine, Seongnam, Korea; ${ }^{29}$ Department of Neurosurgery, Kangbuk Samsung Hospital, Sungkyunkwan University School of Medicine, Seoul, Korea; ${ }^{30}$ Department of Radiation Oncology, Samsung Medical Center, Sungkyunkwan University School of Medicine, Seoul, Korea

\section{REFERENCES}

1. Ostrom QT, Gittleman H, Truitt G, Boscia A, Kruchko C, BarnholtzSloan JS. CBTRUS statistical report: primary brain and other central 
nervous system tumors diagnosed in the United States in 2011-2015. Neuro Oncol 2018;20(suppl_4):iv1-86.

2. Dho YS, Jung KW, Ha J, et al. An updated nationwide epidemiology of primary brain tumors in Republic of Korea, 2013. Brain Tumor Res Treat 2017;5:16-23.

3. Louis DN, Ohgaki H, Wiestler OD, et al. The 2007 WHO classification of tumours of the central nervous system. Acta Neuropathol 2007;114: 97-109.

4. Louis DN, Perry A, Reifenberger G, et al. The 2016 World Health Organization classification of tumors of the central nervous system: a summary. Acta Neuropathol 2016;131:803-20.

5. Louis DN, Perry A, Burger P, et al. International Society Of Neuropathology--Haarlem consensus guidelines for nervous system tumor classification and grading. Brain Pathol 2014;24:429-35.

6. Weller M, Weber RG, Willscher E, et al. Molecular classification of diffuse cerebral WHO grade II/III gliomas using genome- and transcriptome-wide profiling improves stratification of prognostically distinct patient groups. Acta Neuropathol 2015;129:679-93.

7. van den Bent MJ, Klein M, Smits M, et al. Bevacizumab and temozolomide in patients with first recurrence of WHO grade II and III glioma, without $1 \mathrm{p} / 19 \mathrm{q}$ co-deletion (TAVAREC): a randomised controlled phase 2 EORTC trial. Lancet Oncol 2018;19:1170-9.

8. van den Bent MJ, Baumert B, Erridge SC, et al. Interim results from the CATNON trial (EORTC study 26053-22054) of treatment with concurrent and adjuvant temozolomide for $1 \mathrm{p} / 19 \mathrm{q}$ non-co-deleted anaplastic glioma: a phase 3, randomised, open-label intergroup study. Lancet 2017;390:1645-53.

9. Gwak HS, Yee GT, Park CK, et al. Temozolomide salvage chemotherapy for recurrent anaplastic oligodendroglioma and oligo-astrocytoma. J Korean Neurosurg Soc 2013;54:489-95.

10. Nabors LB, Portnow J, Ammirati M, et al. Central nervous system cancers, version 1.2015. J Natl Compr Canc Netw 2015;13:1191-202.

11. Nabors LB, Portnow J, Ammirati M, et al. NCCN guidelines insights: central nervous system cancers, version 1.2017. J Natl Compr Canc Netw 2017;15:1331-45.

12. National Comprehensive Cancer Network. NCCN clinical practice guidelines in oncology, central nervous system cancers. Version 1.2019 (Accessed August 1, 2019, at https://www.nccn.org/professionals/physician_gls/default.aspx\#cns.)

13. Weller M, van den Bent M, Hopkins $\mathrm{K}$, et al. EANO guideline for the diagnosis and treatment of anaplastic gliomas and glioblastoma. Lancet Oncol 2014;15:e395-403.

14. Kim YZ, Kim CY, Lim J, et al. The Korean Society for Neuro-Oncology (KSNO) guideline for glioblastomas: version 2018.01. Brain Tumor Res Treat 2019;7:1-9.

15. Wen PY, Macdonald DR, Reardon DA, et al. Updated response assessment criteria for high-grade gliomas: response assessment in neurooncology working group. J Clin Oncol 2010;28:1963-72.

16. Roh TH, Kang SG, Moon JH, et al. Survival benefit of lobectomy over gross-total resection without lobectomy in cases of glioblastoma in the noneloquent area: a retrospective study. J Neurosurg 2019 Mar 1 [Epub]. Available at https://doi.rog/10.3171/2018.12.JNS182558.
17. Van den Bent MJ. Interobserver variation of the histopathological diagnosis in clinical trials on glioma: a clinician's perspective. Acta Neuropathol 2010;120:297-304.

18. Fuller CE, Schmidt RE, Roth KA, et al. Clinical utility of fluorescence in situ hybridization (FISH) in morphologically ambiguous gliomas with hybrid oligodendroglial/astrocytic features. J Neuropathol Exp Neurol 2003;62:1118-28.

19. Weller M, Pfister SM, Wick W, Hegi ME, Reifenberger G, Stupp R. Molecular neuro-oncology in clinical practice: a new horizon. Lancet Oncol 2013;14:e370-9.

20. Yan H, Parsons DW, Jin G, et al. IDH1 and IDH2 mutations in gliomas. N Engl J Med 2009;360:765-73.

21. Hartmann C, Meyer J, Balss J, et al. Type and frequency of IDH1 and IDH2 mutations are related to astrocytic and oligodendroglial differentiation and age: a study of 1,010 diffuse gliomas. Acta Neuropathol 2009;118:469-74.

22. Eckel-Passow JE, Lachance DH, Molinaro AM, et al. Glioma groups based on $1 \mathrm{p} / 19 \mathrm{q}, \mathrm{IDH}$, and TERT promoter mutations in tumors. $\mathrm{N}$ Engl J Med 2015;372:2499-508.

23. Sanson M, Marie Y, Paris S, et al. Isocitrate dehydrogenase 1 codon 132 mutation is an important prognostic biomarker in gliomas. J Clin Oncol 2009;27:4150-4.

24. Hartmann C, Hentschel B, Wick W, et al. Patients with IDH1 wild type anaplastic astrocytomas exhibit worse prognosis than IDH1-mutated glioblastomas, and IDH1 mutation status accounts for the unfavorable prognostic effect of higher age: implications for classification of gliomas. Acta Neuropathol 2010;120:707-18.

25. Van den Bent MJ, Brandes AA, Taphoorn MJ, et al. Adjuvant procarbazine, lomustine, and vincristine chemotherapy in newly diagnosed anaplastic oligodendroglioma: long-term follow-up of EORTC brain tumor group study 26951. J Clin Oncol 2013;31:344-50.

26. Cairncross G, Wang M, Shaw E, et al. Phase III trial of chemoradiotherapy for anaplastic oligodendroglioma: long-term results of RTOG 9402. J Clin Oncol 2013;31:337-43.

27. Herrlinger U, Tzaridis T, Mack F, et al. Lomustine-temozolomide combination therapy versus standard temozolomide therapy in patients with newly diagnosed glioblastoma with methylated MGMT promoter (CeTeG/NOA-09): a randomised, open-label, phase 3 trial. Lancet 2019; 393:678-88.

28. Lombardi G, De Salvo GL, Brandes AA, et al. Regorafenib compared with lomustine in patients with relapsed glioblastoma (REGOMA): a multicentre, open-label, randomised, controlled, phase 2 trial. Lancet Oncol 2019;20:110-9.

29. Louis DN, Aldape K, Brat DJ, et al. Announcing cIMPACT-NOW: the consortium to inform molecular and practical approaches to CNS tumor taxonomy. Acta Neuropathol 2017;133:1-3.

30. Louis DN, Giannini C, Capper D, et al. cIMPACT-NOW update 2: diagnostic clarifications for diffuse midline glioma, H3 K27M-mutant and diffuse astrocytoma/anaplastic astrocytoma, IDH-mutant. Acta Neuropathol 2018;135:639-42.

31. Killela PJ, Pirozzi CJ, Reitman ZJ, et al. The genetic landscape of anaplastic astrocytoma. Oncotarget 2014;5:1452-7. 